\title{
Fused filament fabrication: comparison of methods for determining the interfacial strength of single welded tracks
}

\author{
Anselm Heuer ${ }^{1,{ }^{*}}$ (D), Jonas Huether ${ }^{2}$ (D), Wilfried V. Liebig ${ }^{1}$ (D), and Peter Elsner ${ }^{1,3}$ \\ ${ }^{1}$ Karlsruhe Institute of Technology, Institute for Applied Materials - Materials Science and Engineering (IAM-WK), \\ Kaiserstrasse 12, 76131 Karlsruhe, Germany \\ 2 TU Dortmund University, Department of Spatial Planning, August-Schmidt-Straße 10, 44221 Dortmund, Germany \\ ${ }^{3}$ Fraunhofer Institute for Chemical Technology (ICT), Joseph-von-Fraunhofer-Straße 7, 76327 Pfinztal, Germany
}

Received: 29 September 2021 / Accepted: 29 November 2021

\begin{abstract}
The mechanical properties of plastic-based additively manufactured specimens have been widely discussed. However, there is still no standard that can be used to determine properties such as the interfacial strength of adjacent tracks and also to exclude the influence of varying manufacturing conditions. In this paper, a proposal is made to determine the interfacial strength using specimens with only one track within a layer. For this purpose, so-called single-wall specimens of polylactide were characterised under tensile load and the interfacial area between the adjacent layers was determined using three methods. It turned out that the determination of the interfacial area via the fracture surface is the most accurate method for determining the interfacial strength. The measured interfacial strengths were compared with the bulk material strength and it was found that the bulk material strength can be achieved under optimal conditions in the FFF process. It was also observed that with increasing nozzle temperature, the simultaneous printing of specimens influences the interfacial strength. To conclude, this method allows to measure the interfacial strength without superimposing the influence of voids. However, for example, the interfacial strength within a layer cannot be determined.
\end{abstract}

Keywords: Fracture surface / bulk material strength / oozing and stringing / pore

\section{Introduction and motivation}

Additive manufacturing has its origin in the so-called rapid prototyping, a technique used to create realistic models of components without the need of manufacturing tools. Nowadays, 3D printers for polymers are easily available and additive manufacturing has become a widely used production technique. Not only the market but also the quality, the mechanical properties and the reliability of additive manufactured structures have grown. Yet, the correlation of printing parameters and mechanical behaviour are not fully understood. Meanwhile, mechanical properties of polymers manufactured by additive manufacturing have widely been discussed. Several parameters are believed to have an effect on the mechanical properties and many have been evaluated scientifically: manufactured structures containing more than a single deposited track will inevitably form voids between individual tracks due to the round geometry of the tracks. Size and nature of these voids will affect stiffness and strength of the printed polymer. This effect has, for

\footnotetext{
* e-mail: anselm.heuer@kit.edu
}

instance, been discussed by Rodríguez et al., mechanical, stating that the most densely packed structures yield higher strength and modulus [1]. Yet, strength of the bulk material was not reached. Besides the effects of extrusion width, nozzle temperature, the orientation of extruded tracks and the colour of the filament, voids between extruded tracks were confirmed to be a significant parameter for material properties in Ahn et al. [2]. Akhoundi et al. effect has also confirmed the effect of the voids, where the infill was investigated in detail [3]. Another influence on the mechanical properties is the orientation of the extruded tracks. Materials with an track orientation along the load direction exhibit the highest strength an modulus $[2,4,5]$. It is believed that this effect occurs due to the two factors: (1) the void content is higher in the direction perpendicular to the load direction. (2) The influence of welded joints between the extruded tracks differs. Whereas welding occurs in all printed materials, they are hardly loaded when the specimen is tested in the same direction as the extruded tracks are oriented but strongly strained when tested perpendicularly. According to Es-Said et al., the penetration of molecular polymer chains is low between adjacent extruded tracks due to low diffusion activity during the welding process [5]. 


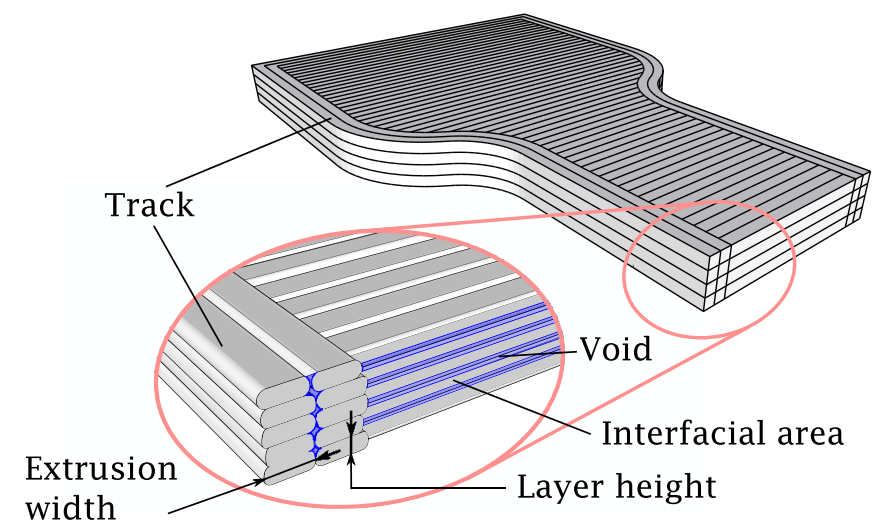

Fig. 1. Position of voids and interfacial areas in specimens with multiple tracks and layers.

Thus, welded joints withstand lower loads than the bulk material itself. The effects on strength and modulus are distinctively pronounced for the directions of $0^{\circ}$ and $90^{\circ}$, respectively. All other angles of loading and orientation can be understood as an overlap of the effects. The disparity of strength and modulus are a consequence of variations of the track orientation within each printed layer. In addition, welding tracks occur in the build-up direction and again, the strength is thereby reduced compared to bulk material [6]. Here, the strength in build-up direction was even less than the in-plane-strength, loaded in $90^{\circ}$. A possible explanation is provided by variations in cooling time. Usually, the time difference between printing two adjacent layers is higher than between two adjacent tracks. Thus, the extruded material will be cooled more and the total heat aiding diffusion processes is reduced. In general, three phases of welding can be defined: the contact of adjacent tracks, mutual wetting without entangling polymer chains (neck growth) and interfacial molecular diffusion [7]. In general, the strength of welding for polymers follows a temperature-time superposition [8]. The longer adjacent tracks or layers are in contact at elevated temperatures, the stronger the emerging interface. Thus, parameters such as the nozzle temperature or the cooling rate of the ventilator affect the welding process. If the same conditions applied to all tracks, then the interfacial strength would theoretically be the same, no matter if the individual tracks lie within a layer or are printed on top of each other. Yet in reality, this is not the case. Therefore, the specimen geometry will affect the mechanical properties determined in experiments. García-Domínguez et al., who compared different test standards, demonstrated this effect [9]. They noted that no standard considering additive manufacturing effects is active.

In addition to the varying heat distribution due to different specimen geometries, bone-shaped, rod-shaped or cuboidal specimens contain different void distributions. Figure 1 depicts interfacial areas and voids in a standard bone-shaped specimen. A variation of printing parameters like e. g. nozzle temperature therefore not only influences the interfacial strength but also the void sizes, which can be explained as follows: Increasing the nozzle temperature decreased the viscosity of the polymer. As FFF-printers are

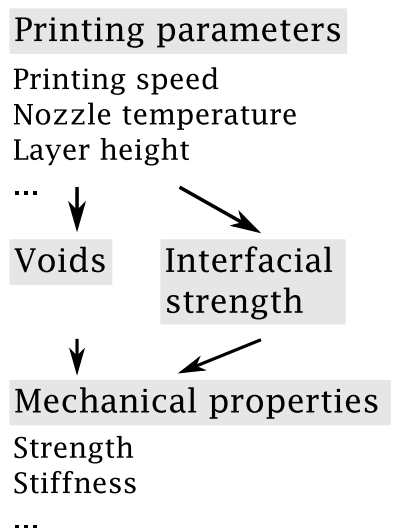

Fig. 2. Simple model how the printing parameters interact with the mechanical properties.

not actively controlled, more polymer is extruded [10]. Thus, the specimen is filled more densely and the size of voids is reduced [11], leading to a potential increase in tensile strength. In addition, more heat is available for welding processes and thus, a stronger interfacial strength is created, again leading to an increased tensile strength. Kuznetsov et al. increasing stated that the separation of effects and their magnitude is not feasible in bulk specimens [11]. Furthermore, higher nozzle temperatures cause increased leakage (oozing) and loss of materials (stringing) as the nozzle follows unsteady discontinuous tracks [12]. Thus, when a standard specimen consisting of several tracks and layers is used for material testing, different effects cannot be separated. If focussing on a single parameter (for instance nozzle temperature) to study or to improve material properties, one might not be able to gain an integral understanding of the process if using bulk specimens. As shown in Figure 2, an improved understanding of the underlying mechanisms can be obtained by linking the printing parameters to the mechanical properties through their effects on the microstructure.

To investigate effects on the printing quality in detail, the material characterisation needs to be able to distinguish different microstructural properties. On the one hand, to investigate the effect of voids, the specimen has to 


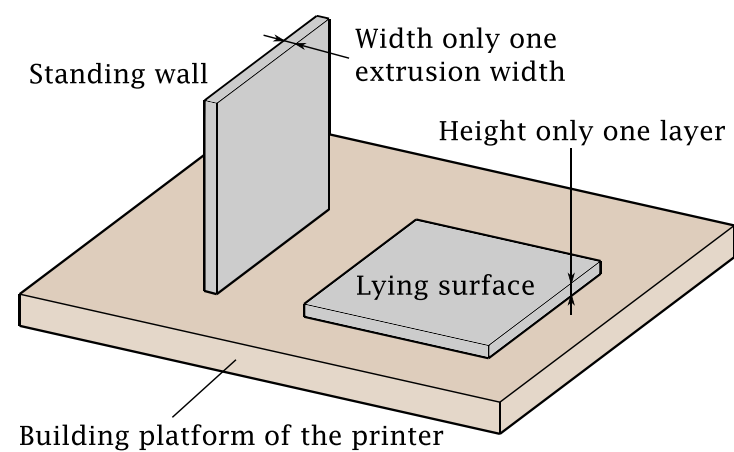

(a)

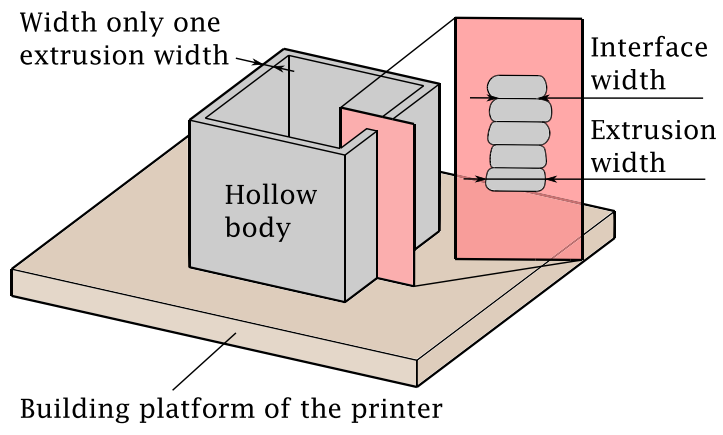

(b)

Fig. 3. Presentation of different types of test shapes for the determination of the interfacial strength. a: Test shapes without parallel tracks or parallel layers and therefore without voids. b: Test shape without containing parallel tracks.

contain adjacent tracks and three dimensional specimens as the one shown in Figure 1 can be used. On the other hand, when only interested in the interfacial strength, it might be beneficial to test an one-layer, foil-like specimen to ensure that an individual interface is investigated, no interaction between layer occur and effects of voids are excluded. Here, the thickness of the specimen is given by the height of a single printed layer, as shown in Figure 3a. Figure 3a also represents a single standing wall that cannot be printed due to stability issues. This sample shape would actually be more appropriate, as the first layers of a printed object are affected by the building platform of the printer and are not representative [13]. A hollow body as that shown in Figure 3b is a reliable and effective alternative.

Such bodys were used for investigations before, for instance by Coogan et al. to obtain the strength between layers, defined as bonding strength by the authors [14]. There, a cuboid as shown in Figure 3b was manufactured and specimens were prepared out of it. The wall thickness was that of a single printed track - i.e. the extrusion width. Coogan et al. also used this specimens to simply determine the extrusion width and the interface width. Despite the fact that both quantities were correlated, the interfacial area was only determined using the extrusion width and thereby the interfacial strength was calculated. A similar approach is found in Han et al. to measure interfacial strength and how to increase it by laserheating the preceding layer [15]. The ratio of extrusion width and interface width was used to calculate the interfacial strength. However, this can only be seen as an approximation to the interfacial area and thus to the interfacial strength. Other researchers referred to the method shown in Coogan et al. to investigate non-planar printing tracks used to increase the specimens isotropy or for a more detailed understanding of printing parameters on strength an modulus $[16,17]$. A thermal simulation of the manufacturing process of such hollow bodys was run and verified experimentally in Lepoivre et al. [18].

Thus, it was proven before that specimens from hollow bodies alike those shown in Figure $3 \mathrm{~b}$ are applicable to investigate and simulate effects in polymer printing. Yet, theses studies did not address the method of interfacial strength measurement itself in detail but focused on the application of these specimens. A uniform procedure specifically for additively manufactured specimens, similar to a standard, would be helpful to make the results more comparable. For example, Fang et al. noted that the ratio between extrusion width and interface width can vary depending on the printing parameters [17]. If only the extrusion width is used to determine the interfacial area, this could have an unfavourable effect on the validity of an investigation. As a consequence, the focus of the work at hand is a profound insight into the application of single-wall specimens and how to minimise measuring errors and effects of the specimen geometry. Furthermore, it should be investigated how reliable the determination of the interfacial area using the extrusion width is. For these investigations, specimens were taken from hollow bodies shown in Figure 3b and printed by using a typical FFF-material. The study investigates various welding temperatures and welding times. In addition, the effect of several simultaneously printed bodies on the interfacial strength is addressed.

\section{Material and methods}

\subsection{Material and devices used}

All specimens were manufactured by using a FFF-type printer, which was developed and configured at the Institute for Applied Materials. This printer is equipped with a direct extruder and the hotend V6 All-Metal HotEnd from E3D. The brass nozzle has a diameter of $0.4 \mathrm{~mm}$ and an annular gap nozzle is installed around the nozzle for cooling. The machine is controlled by the Duet 2 Ethernet board with the firmware RepRapFirmware 2.05. As common for all commercially available printers, material flow is not closed loop controlled.

Polylactide (PLA) was used for all investigations as it is the most common material in FFF-printing and meets all demands of the proposed experimental study. A PLAfilament type BASF Ultrafuse with a recommended printing temperature range of $210^{\circ} \mathrm{C}$ to $230^{\circ} \mathrm{C}$ and glass transition temperature of $60^{\circ} \mathrm{C}$ was used. A diameter of $2.85 \mathrm{~mm}$ was chosen to suit the extruder in use. 
Table 1. Slicer parameters used for printing cuboids.

\begin{tabular}{lc}
\hline Slicer parameter & Setting \\
\hline Layer height & $0.3 \mathrm{~mm}$ \\
Extrusion width & $0.5 \mathrm{~mm}$ \\
Printing and travel speed & $30 \mathrm{~mm} / \mathrm{s}$ and $100 \mathrm{~mm} / \mathrm{s}$ \\
Platform temperature & $60{ }^{\circ} \mathrm{C}$ \\
Nozzle temperature & $190{ }^{\circ} \mathrm{C}, 210^{\circ} \mathrm{C}$ and $230{ }^{\circ} \mathrm{C}$ \\
Cooling of print & Permanent on $100 \%$ power \\
Retraction distance and speed & $2 \mathrm{~mm} \mathrm{by} 40 \mathrm{~mm} / \mathrm{s}$ \\
\hline
\end{tabular}

\subsection{Specimen manufacturing and preparation}

Hollow cuboids open at the top and bottom were printed to investigate the method and determine the interfacial strength. Layer by layer, quadratic fringes were printed one on top of each other. The seam was at the same corner for each layer. As a consequence, the cooling time between printing of two adjacent layers is defined by the time needed to complete a layer. The cuboid was first designed in CAD software with edge lengths of $22 \mathrm{~mm}$, a wall thickness of $0.5 \mathrm{~mm}$ and a height of $60 \mathrm{~mm}$. It was then converted to G-code in the slicer software PrusaSlicer 2.2.0. The slicer settings are listed in Table 1.

To increase the economic efficiency of FFF printing, several components are commonly printed simultaneously. However, this might affect quality and properties of the printed components. To investigate the effects of simultaneous printing, one, two, three, four or five individual cuboids were printed in one process. Leaping from one cuboid to the next, the cooling time of each layer of each cuboid is varied and effects hereof can be studied. Commonly, a so-called retract is used when leaping from one object to the next. The settings of the retract were following the recommendations given for the V6 hotend from E3D. Figure 4 illustrates the printing of one or more cuboids. The individual tracks and the travel paths can be seen. The nozzle temperature was varied in three steps $190{ }^{\circ} \mathrm{C}, 210^{\circ} \mathrm{C}$ and $230^{\circ} \mathrm{C}$ and the different amount of cuboids resulted in cooling times of $3.2 \mathrm{~s}, 9.2 \mathrm{~s}, 13.7 \mathrm{~s}, 18.5 \mathrm{~s}$ and $23.4 \mathrm{~s}$. In summary, the influence of the nozzle temperature, the cooling time and simultaneous printing was investigated with the experimental method. This led to 15 different parameter groups, for each of which twelve specimens were produced. Four specimens could be extracted from one cuboid. Room temperature and humidity were logged before and during the printing process in order to identify external influences.

In a study by Coogan et al. it was shown that no significant differences in strength between punched and laser cutter-cut specimens occurs [14]. Thus, four boneshaped specimens were punched with high speed from each cuboid as this was the most productive procedure to cut specimens. They are hereafter labelled single-walled specimens (SWS). Based on DIN EN ISO 527-1, the SWS had a length of $50 \mathrm{~mm}$, a breadth of $10 \mathrm{~mm}$ in the clamping area and a breadth of $8 \mathrm{~mm}$ in the measuring area (hereinafter referred to as the breadth of the SWS), as shown in Figure 5. The SWS were designed to most likely
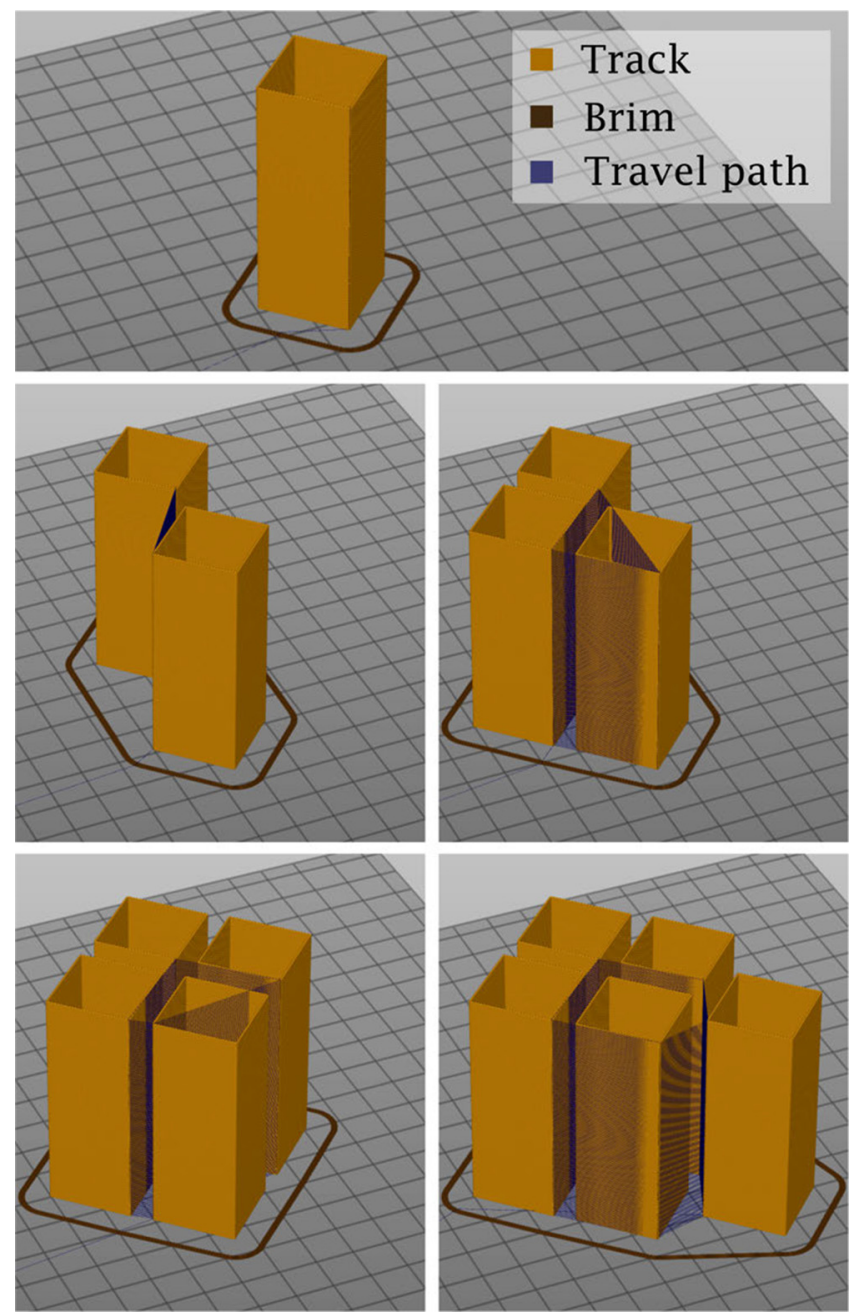

Fig. 4. Representation of the tracks and travel paths in the slicer PrusaSlicer. With only one cuboid, no travel paths are required. For all other constellations, travel paths are present and these partially intersect the tracks. The brim ensures that the material flow is set correctly at the start of printing.

fail in the measuring area. Each cuboid was highly aligned with the punching tool to ensure that the punching edges were perpendicular to the layer interfaces. To determine the bulk material strength of PLA, strands were extruded at temperatures of $190^{\circ} \mathrm{C}, 210^{\circ} \mathrm{C}$ and $230^{\circ} \mathrm{C}$. The feed rate was set to correspond to a printing speed of $30 \mathrm{~mm} / \mathrm{s}$. 


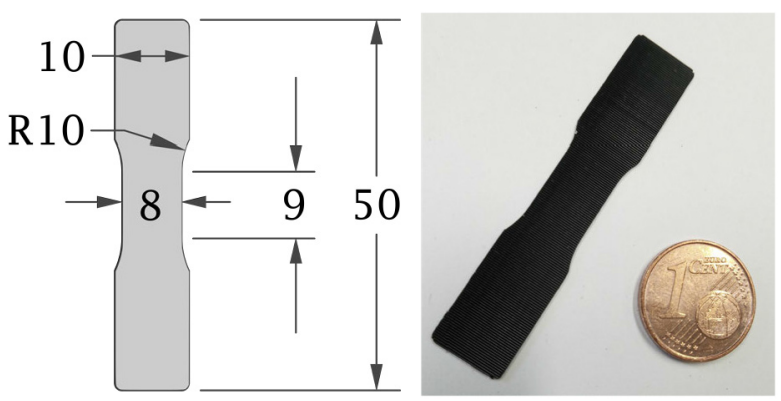

Fig. 5. Geometric dimensions of the single-walled specimens (SWS). All dimensions are in mm. A real specimen is shown next to it for size comparison.

\subsection{Determination of interface area}

The interfacial area needed for determining the interfacial strength is the product of the interface width and the breadth. Coogan et al. discovered that the interface width can vary along the breadth [14]. Consequently, the interface width along the breadth would have to be known in order to determine the exact interface by integration. Fang et al. investigated micro computed tomography scans $(\mu \mathrm{CT})$ of SWS with a resolution of $4.87 \mu \mathrm{m} / \operatorname{voxel}[17]$. This would allow the determination of the interface area with an accuracy given by the $\mu \mathrm{CT}$ s. However, the procedure is time-consuming. Furthermore, the resolution is decreased with increasing specimen size and is thus only applicable for small-sized specimens.

Consequently, approximation procedures are needed. The simplest approximation is multiplying the set extrusion width with the breadth of the SWS. Usually, the set extrusion width does not coincide with the real extrusion width $[14,15,17]$. Both can be harmonised but the corresponding calibration procedure has to be run for each operating point [17]. At this point it is worthwhile to measure the extrusion width with a micrometer. This procedure will hereafter, in accordance with Figure 6, be referred to as interface area measurement through the extrusion width (IAM/E). In general, this is a common procedure to determine the geometry of test specimens.

The second approximation procedure is measuring the interface width by means of a microscope or macroscope focussing on the punched edge an the breadth by means of a micrometer. An extension and improvement is given by measuring each individual interface width prior to testing an later considering the interface that actually failed, labelled as interface area measurement through the interface width (IAM/I) and shown in Figure 6. If for different representative samples with the interface width $w_{\mathrm{i}}$ and the extrusion width $w_{\mathrm{e}}$ the ratio $R$ is calculated according to

$$
R=\frac{w_{i}}{w_{e}} \text { with } R \neq f(\text { breadth, parameter space), }
$$

the determination of the interface according to IAM/E can be improved. In this case, the extrusion width of a specimen is measured and ratio $R$ is used to calculate the interface

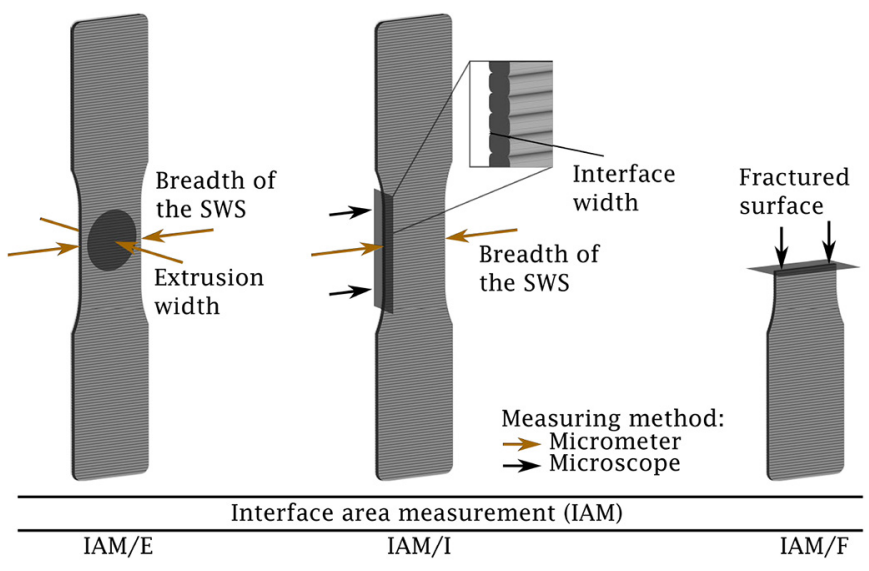

Fig. 6. Graphical explanation of the interface area measurement (IAM) methods IAM/E, IAM/I and IAM/F. With the IAM/E method, only a micrometer screw is used. The circle corresponds approximately to the diameter of the micrometer screw's measuring spindle. For IAM/I, a macroscope is additionally used to determine the interface width. IAM/E and IAM/I are carried out before the tensile test, while for IAM/F the fracture surface is measured directly after the tensile test.

width. However, this requires a constant ratio $R$ along the breadth and the parameter space, otherwise this conversion leads to additional measurement errors.

A third approximation would be to assume that interfacial area and fractured surface are the same, hereafter labelled interface area measurement through the fractured surface (IAM/F) and also shown in Figure 6. Here, the fractured surface is measured subsequently to testing by means microscope or macroscope.

In the work at hand, all there approximations are used and compared: extrusion widths and breadths were measured with a micrometer, all interface widths and the fractured surface with a macroscope. The fractured surface was investigated with the software Fiji using a tetragon. Subsequent to testing, all layers were counted to identify the fracture surface and to relate it to the measured interface width.

For determining the material's tensile strenght, the load at failure and the cross-sectional area of the extruded strands are needed. It was assumed that they approximate a perfect circular shape. The diameter was gauged with a micrometer prior to testing.

\subsection{Procedure to investigate the interfacial strength}

Tensile test of the punched SWS were run on a universal testing device Zwick Z2.5 by Zwick/Roell with a $1 \mathrm{kN}$ load cell. The grippers were coated with rubber to prevent specimen failure caused by squeezing. Specimens that failed within the gripping region were excluded from the experimental study. All specimens were gripped $15 \mathrm{~mm}$ on both ends and were tested at a speed of $3 \mathrm{~mm} / \mathrm{min}$ with an initial load of $1 \mathrm{~N}$. Extruded strands were tested with the same device but using a modified setup: a $50 \mathrm{~N}$ load cell was 


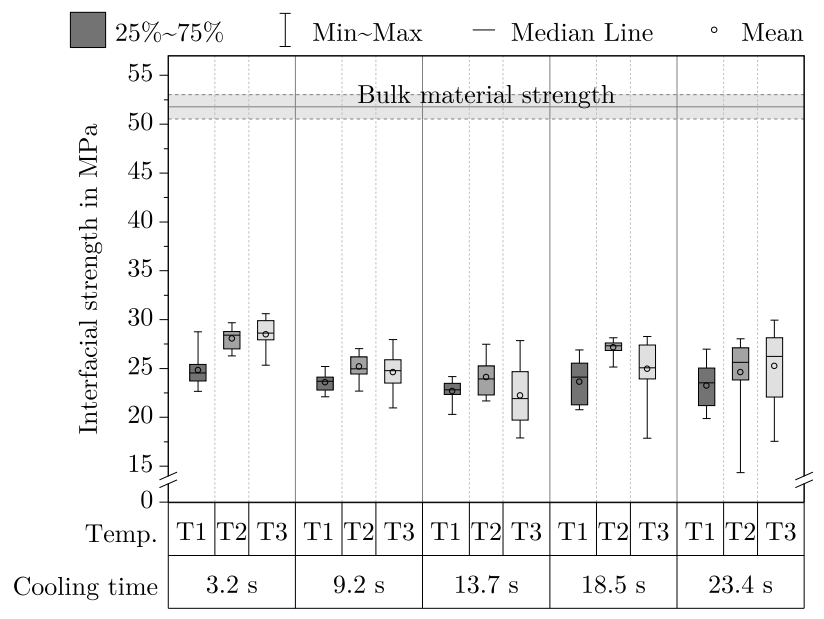

(a)

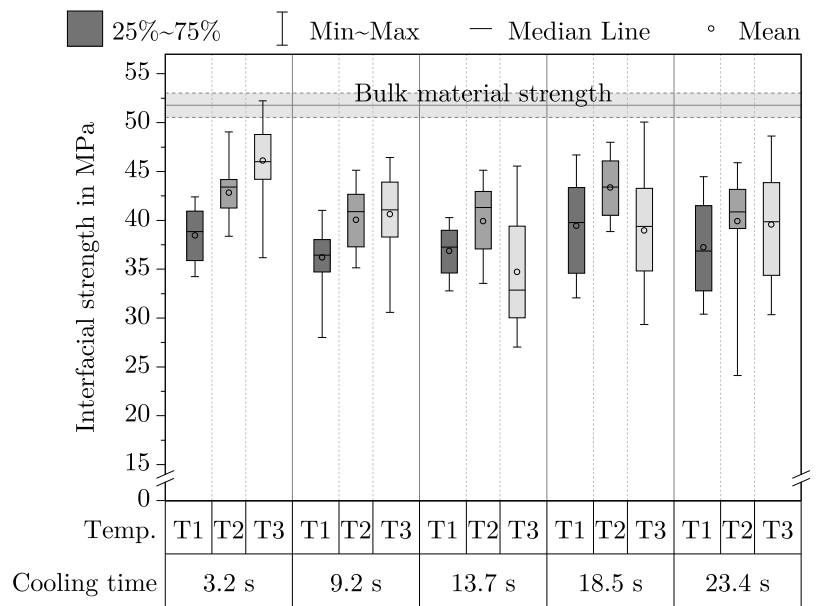

(b)

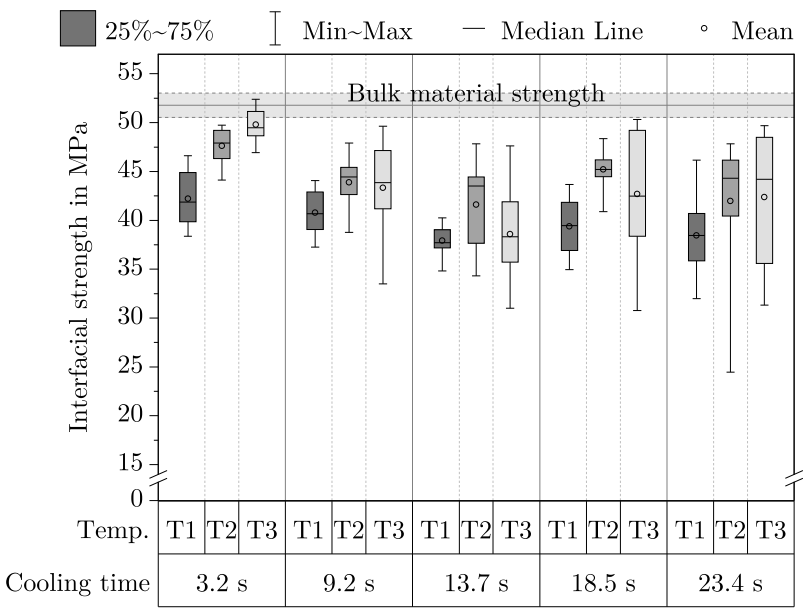

(c)

Fig. 7. Influence of nozzle temperature $\left(\mathrm{T} 1=190{ }^{\circ} \mathrm{C}, \mathrm{T} 2=210^{\circ} \mathrm{C}, \mathrm{T} 3=230{ }^{\circ} \mathrm{C}\right)$ and cooling time $(3.2 \mathrm{~s}, 9.2 \mathrm{~s}, 13.7 \mathrm{~s}, 18.5 \mathrm{~s}, 23.4 \mathrm{~s})$ on interfacial strength. A distinction is made between the three methods (a) IAM/E, (b) IAM/I and (c) IAM/F. To classify the interfacial strengths, the bulk material strength (solid line) is shown with its standard deviation (dashed lines). Mean values and the scatter of the interfacial strengths differ depending on the method considered.

used and grippers controlled with pressurised air and optimised for fibre testing were used. This setup and the grippers were originally designed for testing single fibres of carbon or glass and are described in detail in [19]. In the work at hand, the device was used to determine tensile strength but no optical strain measurements were run. Testing speed was set to $2 \mathrm{~mm} / \mathrm{min}$ in order to reach the load at failure after approximately $60 \mathrm{~s}$. The initial load was set to $0.02 \mathrm{~N}$. Both experiments were run at the same circumstances in an air-conditioned test facility.

\section{Results}

The interfacial strengths, based on the tensile tests and determining the cross-sectional areas in the three aforementioned procedures (IAM/E, IAM/I, IAM/F), are shown in Figure 7 . The graph represents the interfacial strength for different parameter groups consisting of nozzle temperature and cooling time. Boxplots were chosen to to identity statistical variations. The mean value of the material strength and its standard deviation are shown as solid and dashed lines, respectively. With increasing nozzle temperature, the interfacial strength is increased. This effect is seen for all cooling times, however, for cooling times higher than $9.2 \mathrm{~s}$, the group of parameter $230^{\circ} \mathrm{C}$ no longer follows this trend. To test for significance, an analysis of variance (ANOVA) was conducted with all groups. The effect of the nozzle temperature was found to be highly significant for all cooling times and all three interface measurement methods $(\alpha<0.001)$. Furthermore, the cooling time between $3.2 \mathrm{~s}$ and $9.2 \mathrm{~s}$ has a highly significant influence on the interfacial strength for all three temperatures and interfacial measurement methods. In this case, the interfacial strength decreases with increasing cooling time. If the significance is determined within all groups of $190{ }^{\circ} \mathrm{C}$ and $210^{\circ} \mathrm{C}$ and a cooling time higher $9.2 \mathrm{~s}$, no significant influence can be found for the interfacial 
Table 2. Comparison of the standard deviations between the different methods for measuring the interfacial area and different nozzle temperatures. To determine the standard deviations, an average was taken over the cooling time.

\begin{tabular}{llll}
\hline Interface area measurement (IAM) method & \multicolumn{3}{c}{ Nozzle temperature } \\
\cline { 2 - 4 } & $190^{\circ} \mathrm{C}$ & $210^{\circ} \mathrm{C}$ & $230^{\circ} \mathrm{C}$ \\
\hline $\mathrm{IAM} / \mathrm{E}$ & $1.74 \mathrm{MPa}$ & $2.11 \mathrm{MPa}$ & $2.84 \mathrm{MPa}$ \\
$\mathrm{IAM} / \mathrm{I}$ & $3.89 \mathrm{MPa}$ & $3.92 \mathrm{MPa}$ & $5.36 \mathrm{MPa}$ \\
$\mathrm{IAM} / \mathrm{F}$ & $2.78 \mathrm{MPa}$ & $3.90 \mathrm{MPa}$ & $5.11 \mathrm{MPa}$ \\
\hline
\end{tabular}

measurement methods IAM/I and IAM/F, respectively. Yet, a significant effect is seen for IAM/E $(\alpha<0.05)$. For the non-significant case, this means that the null hypothesis, no influence of prolonged cooling on the interfacial strength, must not be rejected. The interfacial strengths of IAM/F are on average about $71 \%$ higher than those of IAM/E, while they are about $7 \%$ higher than those of IAM/I.

The magnitude of the standard deviations within the groups differs between the parameter groups and between the three interfacial measurement methods, as shown in Table 2. Thus, the standard deviations of the interfacial strength are lowest for IAM/E, while IAM/I has the highest standard deviations. Those for IAM/F are slightly below IAM/I. Regardless of the interface measurement methods, the standard deviations of the groups with $190{ }^{\circ} \mathrm{C}$ are the lowest compared to the other groups. The standard deviations increase in the groups of $210^{\circ} \mathrm{C}$ and even more prominent in the groups of $230^{\circ} \mathrm{C}$.

By means of testing extruded single strands, the bulk material strength of PLA was measured and is shown as a function of nozzle temperature in Figure 8. No significant difference was measured between the $190^{\circ} \mathrm{C}$ and $210^{\circ} \mathrm{C}$ groups, while an evaluation with all three groups predicts a significant influence of the nozzle temperature. The mean and standard deviation of the bulk material strength used in Figure 7 were calculated from all three groups. The mean value is $51.8 \mathrm{MPa}$ and the standard deviation is $1.3 \mathrm{MPa}$.

\section{Discussion}

\subsection{Evaluation of the interface measurement methods}

When using method IAM/E, the extrusion width is measured and the corresponding interfacial areas are expected to be far higher than those determined for IAM/I and IAM/F. Less difference is expected between IAM/I and IAM/F. Thus, variations between IAM/E, IAM/I and $\mathrm{IAM} / \mathrm{F}$ will be discussed in detail in the following. It was shown before $[14,17,20]$ that the interfacial strength is increased with increased nozzle temperature and decreased cooling time. Applying IAM/I or IAM/F, the effect was shown to be highly significant. As stated in Table 2, the standard deviation is higher for IAM/I. A higher standard deviation might be attributed to the manufacturing process and thus, understanding the standard deviation might help to find production errors. In what follows, the measuring uncertainties are discussed to understand which methods reflects the real standard deviation more reliably. First off, a principle-related measurement uncertainty due

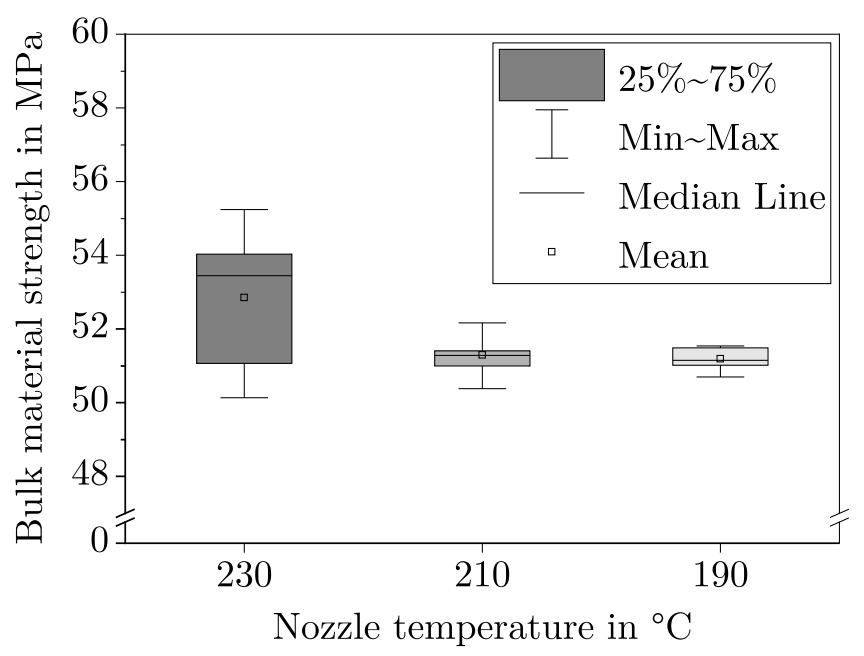

Fig. 8. Influence of nozzle temperature on bulk material strength.

to the different measurement must be mentioned here. By using IAM/I, only one measurement of the interface width is used to approximate the interfacial area. As said in Section 2.3, it may vary along the breadth of the SWS. Investigating a series of images of fractured surfaces, this was confirmed for several specimens and is demonstrated in Figure 9 for two specimens. Furthermore, punching the specimens caused smearing of the edges which resulted in a systematic error. To approximate the value the error, specimens were embedded in resin, ground and polished and compared to original microscopic images. A maximum mismatch of the interfacial areas of $0.33 \mathrm{~mm}^{2}$ was found. However, it is unlikely that all specimens exhibit such a error.

The difference between IAM/I and IAM/F is represented as histogram for all specimens in Figure 10. Here, IAM/I shows a wider distribution of the interfacial area. The mean value of the interfacial area is $2.75 \mathrm{~mm}^{2}$ using IAM/I and $2.57 \mathrm{~mm}^{2}$ by using IAM/F, respectively. Thus the difference between the two mean values is $0.18 . \mathrm{mm}^{2}$. Applying a normal distribution on the aforementioned systematic error regarding the smearing of the edges, the mean deviation is $0.165 \mathrm{~mm}^{2}$, which approximately corresponds to the difference of the the interfacial areas $\left(0.18 \mathrm{~mm}^{2}\right)$ by using IAM/I and IAM/F. Thus, it is assumed that the difference is caused by the systematic error. In addition, the accuracy of both methods is affected by the user, as the edges of the areas are defined manually. 


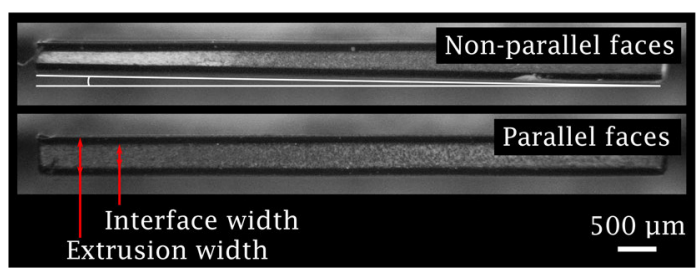

Fig. 9. Fracture surface of one specimens with non-parallel and parallel faces, respectively. Accordingly, the interface width is not constant over the breadth of the SWS.

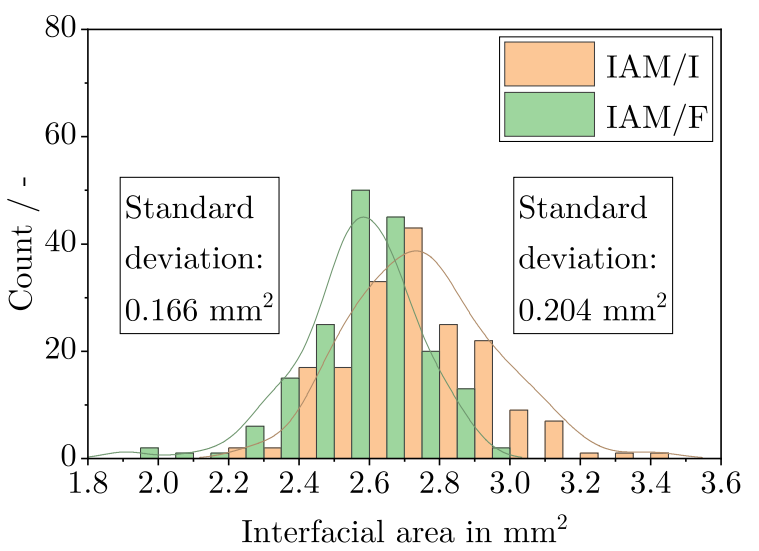

Fig. 10. Distribution of the fracture surface of all specimens for the two methods IAM/I and IAM/F. For a better presentation, a continuous distribution was applied to the discontinuous data with the help of a kernel density estimator. Scatter and mean differ between the methods.

The measuring error is higher for low pixel-ratios. The ratio was $185.5 \mathrm{Pixel} / \mathrm{mm}$ for IAM/I and $147.8 \mathrm{Pixel} / \mathrm{mm}$ for $\mathrm{IAM} / \mathrm{F}$, respectively. The difference in resolution was due to differences in the usable image section. In order to estimate the measurement uncertainty, specimens were measured manually several times and a mean deviation of the interface was determined. For IAM/F this is about $0.13 \mathrm{~mm}^{2}$. The interface width according to IAM/I was measured with a macroscope. The main reason for the measurement uncertainty in IAM2 is the misallocation of pixels. To determine the misallocation, sections of the macroscope images were compared with additional microscope images. It was assessed that the misallocations are up to 8 pixels, resulting in a maximal error of $0.41 \mathrm{~mm}^{2}$. Applying a normal distribution, the mean deviation of $\mathrm{IAM} / \mathrm{I}$ is $0.205 \mathrm{~mm}^{2}$. It is concluded that the wider distribution of IAM/I, as seen in Figure 10, is caused by errors in image analysis.

All methods showed that the effects of nozzle temperature and cooling time until $9.2 \mathrm{~s}$ were be highly significant. However, IAM/E also predicted a significant effect over a cooling time of $9.2 \mathrm{~s}$, which was not verified by either IAM/I or IAM/F. This is not really plausible, because publications on cooling behaviour tend to indicate a few seconds for cooling below glass the transition temperature. For example, Coogan et al. modelled the cooling behaviour of a single printed track for hollow cuboids and fell below glass the transition temperature of $110^{\circ} \mathrm{C}$ after approximately $5 \mathrm{~s}$ for acrylonitrile butadiene styrene (ABS) [20]. Below this temperature, interfacial diffusion of polymer chains takes no longer place. No further increase of interfacial strength is expected [21]. As the glass transition temperature of PLA is $60^{\circ} \mathrm{C}$, diffusion can appear slightly longer. Yet, within a few more seconds no more diffusion is expected. The significance above $9.2 \mathrm{~s}$ could be attributed to a statistical effect: for smaller variations in groups, minor differences between average values become significant. As can be seen in Table 2, the distribution of the interfacial strength for IAM/E is more narrow compared to the other methods. But the decreased distribution does not reflect the real variations of the interfacial area. By using a micrometer with cylinders of $6.5 \mathrm{~mm}$ in diameter, approximately 22 extrusion widths are covered. This aspect is also illustrated in Figure 6. Yet, an extremum is measured instead of an extrusion width at the fracture. As shown in Figure 11a, the extremum may have nothing to do with either the interface width or the actual extrusion width at the fracture. The scatter of the measured extrusion width according to IAM/E can rather be attributed to minor variations in the position of the tracks. From the measured extrusion width and interface width obtained from IAM/I, the ratio $R$ was calculated and is shown in Figure 11b. It can be seen that the ratio $R$ can vary by 0.15 for the same parameters. In addition, no significant effects on the ratio $R$ can be observed. If the ratio $R$ were constant, then the distribution of the measured extrusion width and the interface width and thus the interfacial area would be the same. Thus, the real distribution of the interfacial areas is not correctly captured by IAM/E and thus effects of the interfacial strength are modified.

Even if the scattering of IAM/E does not appear to be correct, the average interfacial strengths can be more closely approximated to the true interfacial strength by determining the ratio $R$ on some specimens with the help of equation (1) and then converting the measured extrusion width into the interface width. If only the IAM/E method is used, this can help to expose spurious effects on the interfacial strength. Thus Coogan et al. uncovered a decrease in interfacial strength with increased layer height an expected this to be caused by an increase in pressure due to the previous layer [14]. Fang et al. were able to show that ratio $R$ decreased with increased layer height, thus changing the interfacial area and that, as a consequence, the effect on the interfacial strength vanished [17]. Consequently, it cannot be assumed that ratio $R$ is constant for different printing parameters and should be taken into account in IAM/E.

It can be summarized that with IAM/E the scatter of the interfacial strengths are inadmissibly changed and that without the consideration of the ratio $R$ the strengths do not correspond to the interfacial strengths. IAM/E should therefore not be used for a precise determination of the interfacial strengths. 


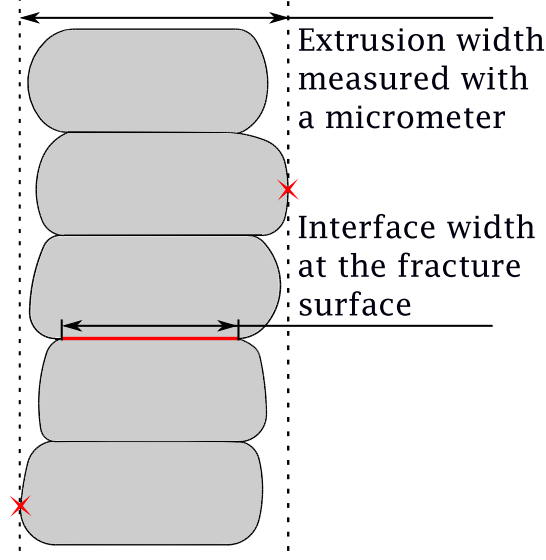

(a)

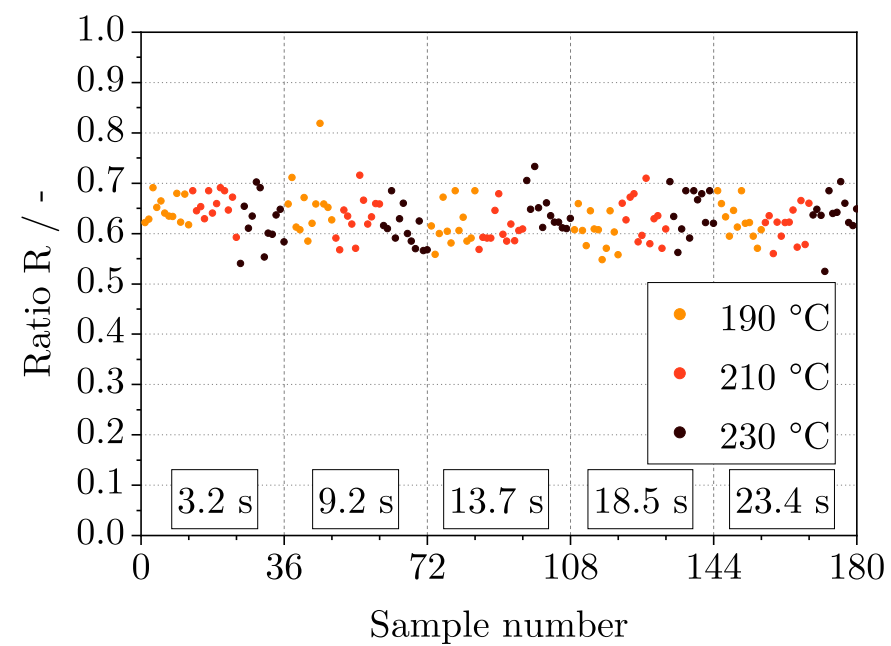

(b)

Fig. 11. Comparison between the extrusion width $w_{\mathrm{e}}$ and the interface width $w_{\mathrm{i}}$. a: If the extrusion width is measured with a micrometer, then a maximum width is actually measured. b: The ratio $R$ for all tested specimens. Each 36 specimens belong to a parameter group with the same cooling time. The nozzle temperature is arranged in ascending order within equal cooling time.

\subsection{Comparison between interfacial strength and bulk material strength}

By determining the interfacial strength on SWS, it could be shown that the weld strength increases significantly with increasing nozzle temperature and decreasing cooling time. This effect was already described by several authors $[11,14,17,20]$. The effects are plausible and the underlying physical theory has been developed in the 1980s for bulk polymers. The theory states that the ends of polymer chains diffuse beyond a weld surface and thus penetrate into each other's polymer base structure [21]. At some point, the micro structure of the welding zone and that of the base material are no longer distinguishable. Mathematically, the weld strength has then reached 1 .

Welding of adjacent layers in the FFF-process can be described by a temperature-time-superposition. Based on the interfacial strengths for different cooling times, it can be recognised in Figure 7 that the influence of the cooling times is difficult to determine. After a few seconds, the increasing cooling time is no longer relevant. Models suggest that this is due to the fast cooling of the track last deposited [20]. When depositing the next track, the internal heat of this track is more effective than the residual heat of the previous track. Postulating that applying the temperature-time-superposition allows for setting the interfacial strength arbitrarily, one might ask whether and with which conditions FFF-processes enables reaching the bulk material strength. This was the focus in a review study by Peterson, stating that some experiments approached the bulk material strength [22]. For instance, Coogan et al., who applied IAM/E and did not correct by the ratio $R$, reached $75 \%$ of the bulk material strength [14]. Adjusting their results by the ratio $R, 85 \%$ were obtained. Considering specimens containing several tracks aligned rectangular to the testing direction, Casavola et al. achieved $21 \%$ of bulk material strength [23]. The low strenght is likely to be caused by neihter considering the actual interfacial area nor voids.

The question of reaching the material strength can only be determined reliably when measuring the bulk material strength and the interfacial strength sufficiently accurate and comparing both. The bulk strength of the PLA used in this study was determined on extruded strands and shows no significant scatter. Yet, it was not expected that the scatter is increased for the specimens produced at a nozzle temperature of $230^{\circ} \mathrm{C}$. It can be deduced that the reduced viscosity caused higher variations of the diameter along the strand, resulting in faulty measurements of the crosssectional area. Despite the increased scatter within the $230{ }^{\circ} \mathrm{C}$ group, the bulk material strength is less prone to variations than the interfacial strength. In order to accurately determine the interfacial strength it is essential to ensure precise determination of the interfacial area. Method IAM/E, which is commonly applied in experimental studies, is not to be relied on for accurate strength measurements, even if the ratio $R$ is considered. This is because the ratio $R$ is susceptible to high scatter, as can be seen in Figure 11b. As discussed in the previous chapter, $\mathrm{IAM} / \mathrm{F}$ is most precise when it comes to determining the interfacial area. When it comes to answering the question of whether the bulk material strength has been achieved, however, uncertainties must also be expected with IAM/F.

If the interfacial strength is close to the strength of the bulk material, then plastic deformation should also occur at the interface, because no failure occurs before plastic deformation begins. For specimens produced at $230{ }^{\circ} \mathrm{C}$ nozzle temperature, curvature of the fracture surface could be observed as shown in Figures 12b and 12c. Specimens at $190{ }^{\circ} \mathrm{C}$ nozzle temperature, on the other hand, showed a flat fracture surface. This is consistent with the material behaviour in the tensile test. In Figure 12a, a plastic range 


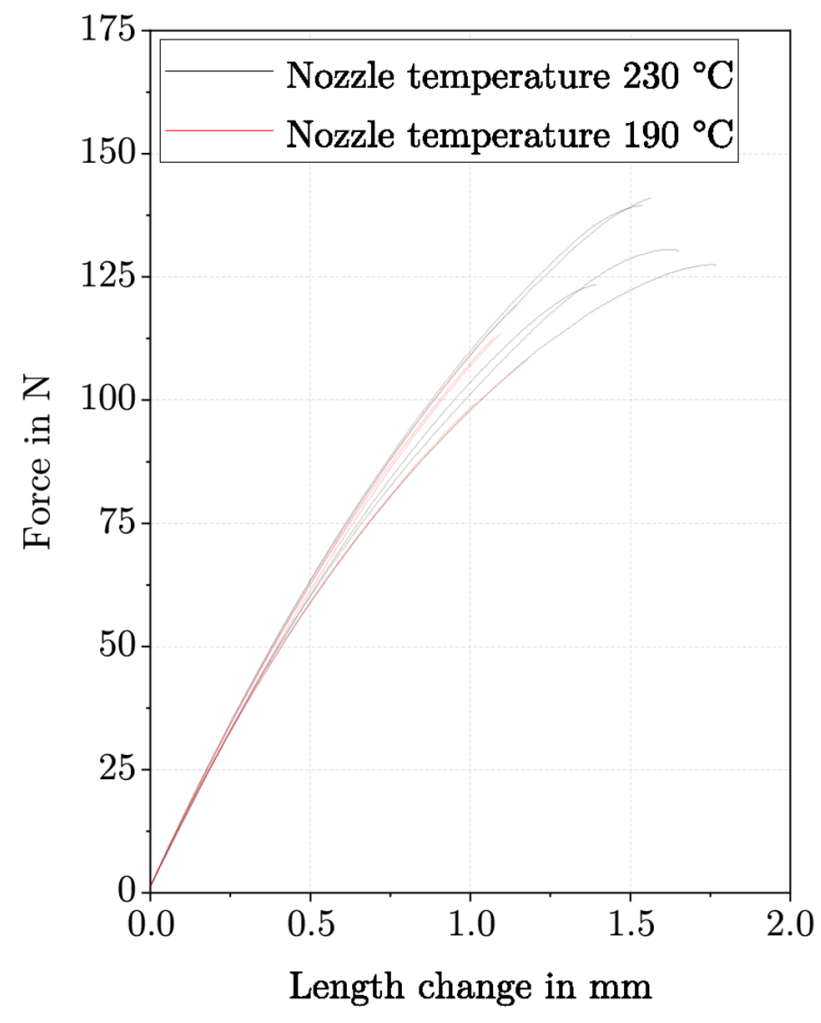

(a)

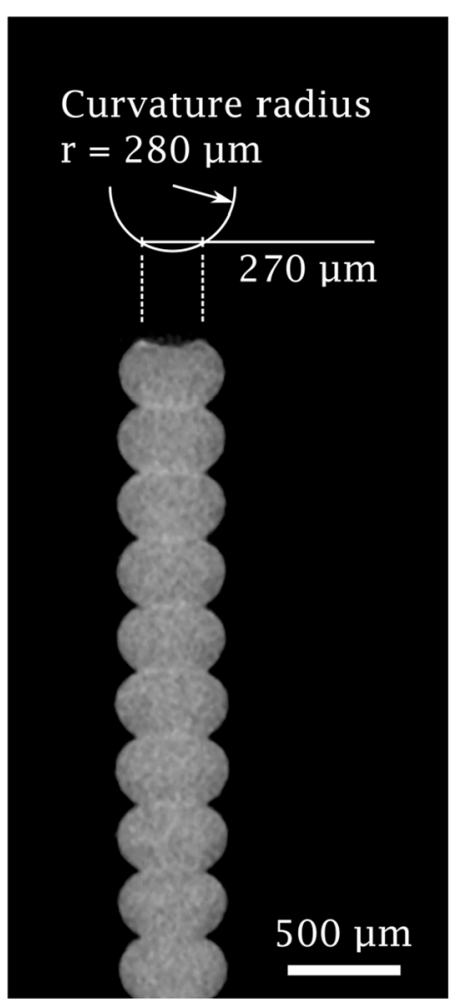

(b)

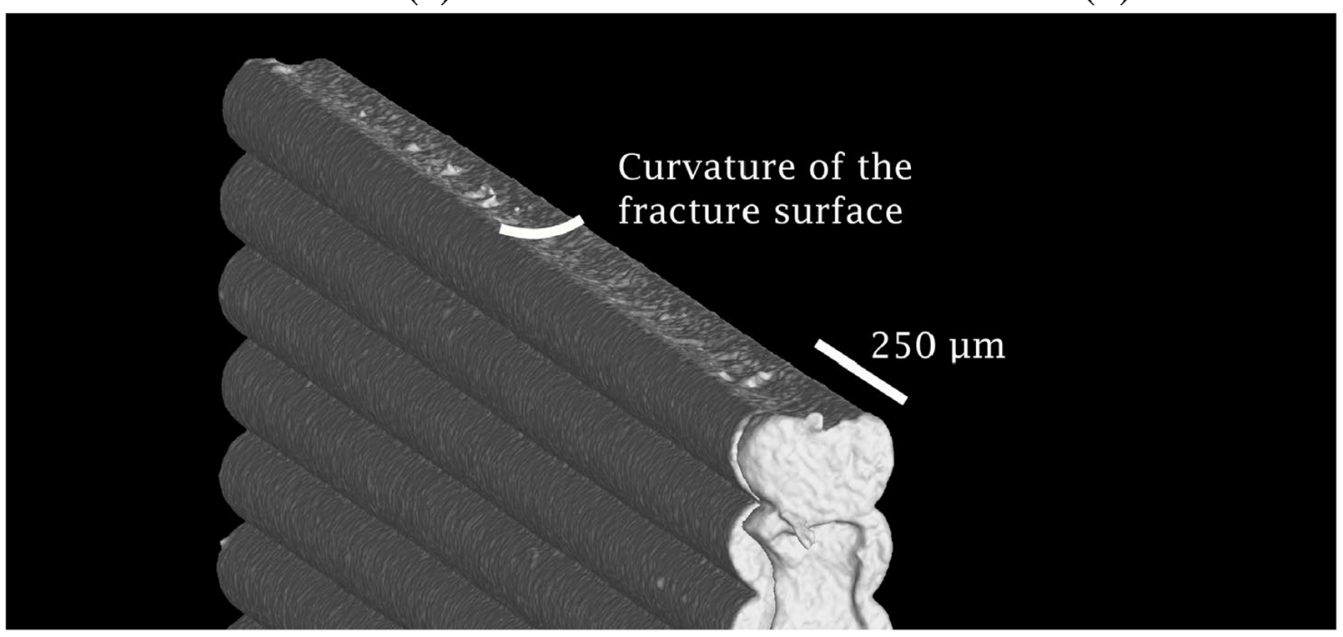

(c)

Fig. 12. All specimens shown had a cooling time of $3.2 \mathrm{~s}$. a: Five curves from the tensile test were each selected as an example. Specimens made at $230^{\circ} \mathrm{C}$ nozzle temperature show a larger change in length compared to $190{ }^{\circ} \mathrm{C}$. Furthermore, the curves at $230^{\circ} \mathrm{C}$ show a plastic deformation range. b: $\mu \mathrm{CT}$ scan of a specimen at $230^{\circ} \mathrm{C}$ nozzle temperature. Curvature of the fracture surface due to deformation after the tensile test. c: 3D render of the specimen from (b). The curvature of the fracture surface remains constant over the breadth of the SWS. 


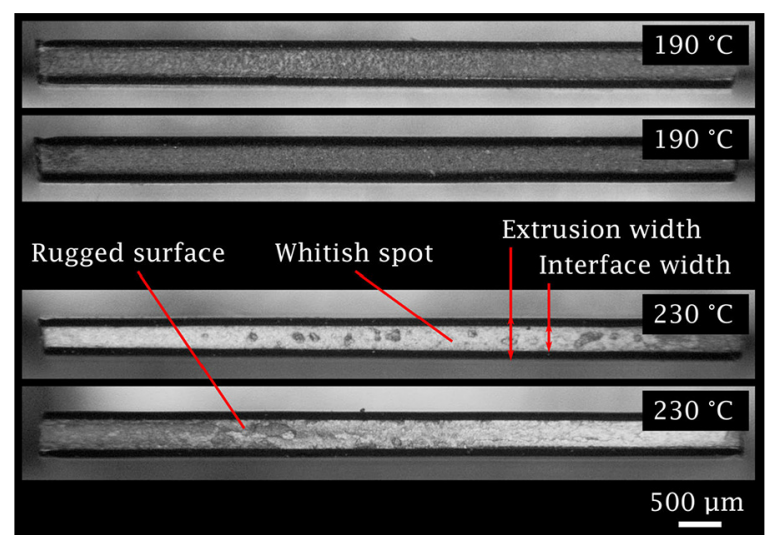

Fig. 13. Comparison of two fracture surfaces of specimens with $230^{\circ} \mathrm{C}$ and $190^{\circ} \mathrm{C}$ nozzle temperature. All specimens shown had a cooling time of $3.2 \mathrm{~s}$. The fracture surfaces of the specimens with $190^{\circ} \mathrm{C}$ nozzle temperature appear homogeneous in colour, whereas the specimens with $230^{\circ} \mathrm{C}$ additionally show whitish spots.

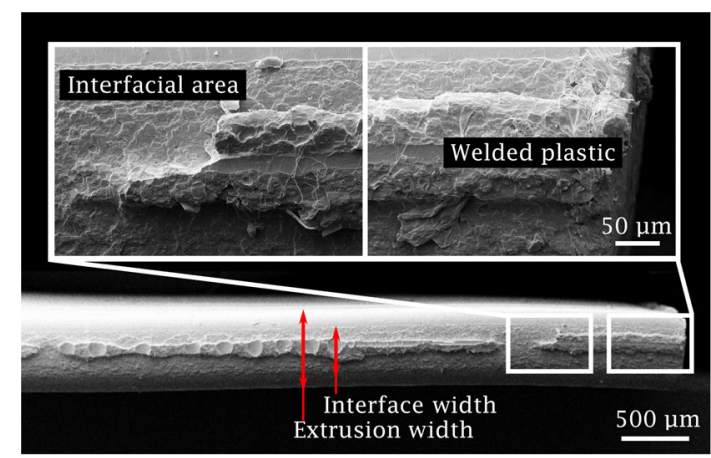

Fig. 14. SEM image of a fracture surface. The cooling time of the specimen was $18.5 \mathrm{~s}$ and the nozzle temperature was $230^{\circ} \mathrm{C}$. Welded parts of the former overlying track remain on the fracture surface. The fracture did not only occur along the interface.

can be determined for specimens with $230^{\circ} \mathrm{C}$ nozzle temperature. In comparison, the specimens at $190^{\circ} \mathrm{C}$ have no plastic range. This leads to an error because according to $\mathrm{IAM} / \mathrm{F}$ the fracture surface is set equal to the original interfacial area. Assuming that the original interfacial area is more like the developed fracture surface than the projected area when viewed from above, an area difference can be calculated. With a curvature radius of $280 \mu \mathrm{m}$ and an averaged interface width of $270 \mu \mathrm{m}$ the original interfacial area would be approximately $4 \%$ larger than the determined value. This results in a systematic overestimation of the interfacial strength of $2 \mathrm{MPa}$ for parameter sets $230^{\circ} \mathrm{C}$ and $3.2 \mathrm{~s}$. At this point it could be mentioned that the specimens did not reach the material strength. But it is unlikely that the interfacial strength reach the bulk material strength as the structured surface of FFF-specimens leads to a significant notch effect. Such a notch effect was investigated and verified by Allum et al. [24]. In addition to the method for determining the interfacial strength, other indications must also be taken into account. Comparing the fractured surfaces in Figure 13 of specimens produced at a nozzle temperature
$230{ }^{\circ} \mathrm{C}$ with those of $190{ }^{\circ} \mathrm{C}$ whitish material is observed. The white colour is caused by crazing and denotes the transition of pure pull-out fracture to crazing fracture, which inhibits increased entangling of polymer chains at the interface [25]. Furthermore, the fractured surfaces of specimens type $230{ }^{\circ} \mathrm{C}$ appears more rugged and material is missing at some spots. This may indicate that some regions of the interface have reached the bulk material strength, so that fracture can occur within the printed track. Here, the interface is no longer the weakest link but crack propagation occurs more arbitrarily. Scanning electron micrographs of several fractured surfaces revealed that parts of tracks printed at $230^{\circ} \mathrm{C}$ still adhered together and that the crack did not propagate along the interface. This can be seen at the specimen in Figure 14. This indicates that the bulk material strength is reached.

Collecting all indications for reaching the material strength and the considering uncertainties of the methods, it can be presumed that specimens of parameter set $230^{\circ} \mathrm{C}$ and $3.2 \mathrm{~s}$ get very close to the bulk material strength. Without taking into account the systematic overestimation due to the curvature of the fracture surface, $96 \%$ of the bulk material strength is reached. Under favourable conditions, it is presumably possible to achieve the bulk material strength at an interface in the FFF process. A reduction in the strength of components consisting of several tracks could in some cases be attributed to voids between the tracks rather than insufficient interfacial strength. This can be shown with sufficient accuracy by using SWS, the $\mathrm{IAM} / \mathrm{F}$ method and, for example, microscope images.

\subsection{Defects caused by oozing and stringing}

Especially for the parameter sets with a nozzle temperature of $230^{\circ} \mathrm{C}$, a greater scattering of the interfacial strengths is observed from the cooling time of $9.2 \mathrm{~s}$ onwards compared to other parameter sets. The average interfacial strength for these specimens is no longer above those at a nozzle temperature of $210^{\circ} \mathrm{C}$, as this can be seen in Figure 7 . In contrast, specimens of the parameter set $230^{\circ} \mathrm{C}$ and $3.2 \mathrm{~s}$ have no increased scatter compared to the specimens at $210^{\circ} \mathrm{C}$ or $190^{\circ} \mathrm{C}$. Evaluation of the fractured surfaces showed that for some specimens above a cooling time of $9.2 \mathrm{~s}$ combined with nozzle temperature $210^{\circ} \mathrm{C}$ and $230^{\circ} \mathrm{C}$, respectively, pores exist on the fractured surface. Table 3 shows that the existence of pores in a parameter group is associated with increased scatter. Figure 15a shows three fracture surfaces with pores. To ensure that these are indeed pores, additional SEM images were taken. On these, the pores in the interface are clearly visible. Investigations on a specimen basis by means of $\mu \mathrm{CT}$ showed that pores did not only occur on fractured surfaces but sometimes also at other interfacial regions. So if a specimen has pores on the fracture surface, then these defects appear again and again in the specimen in a statistically distributed manner.

A comparison of the interfacial strength of specimens with and without pores on the interface shows that the specimens with pores have a lower strength and thus increase the scatter of the group. The difference of the strengths is shown for the specimens of the group $230^{\circ} \mathrm{C}$ and $18.5 \mathrm{~s}$ in Figure 15b. Without the specimens with pores, 
Table 3. For each parameter group, the number of specimens with pores on the fracture surface is given. There were a total of 12 specimens per group. The standard deviation (SD) of the interfacial strength according to IAM/I is also given for each group.

\begin{tabular}{lllllll}
\hline \multirow{2}{*}{ Nozzle temperature } & & \multicolumn{4}{c}{ Cooling time } \\
\cline { 3 - 7 } & & $3.2 \mathrm{~s}$ & $9.2 \mathrm{~s}$ & $13.7 \mathrm{~s}$ & $18.5 \mathrm{~s}$ & $23.4 \mathrm{~s}$ \\
\hline \multirow{2}{*}{$190^{\circ} \mathrm{C}$} & Specimens width pores & 0 & 0 & 0 & 0 & 0 \\
& SD of interfacial strength in MPa & 2.81 & 2.41 & 1.44 & 2.82 & 3.86 \\
$210{ }^{\circ} \mathrm{C}$ & Specimens width pores & 0 & 4 & 5 & 1 & 3 \\
& SD of interfacial strength in MPa & 1.78 & 2.50 & 4.52 & 1.78 & 6.56 \\
$230{ }^{\circ} \mathrm{C}$ & Specimens width pores & 0 & 5 & 6 & 7 & 8 \\
& SD of interfacial strength in MPa & 1.63 & 4.74 & 4.55 & 6.38 & 6.62 \\
\hline
\end{tabular}

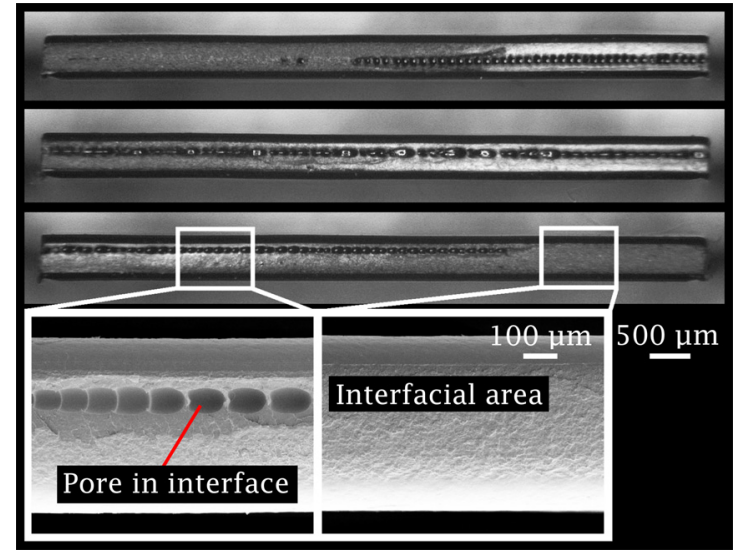

(a)

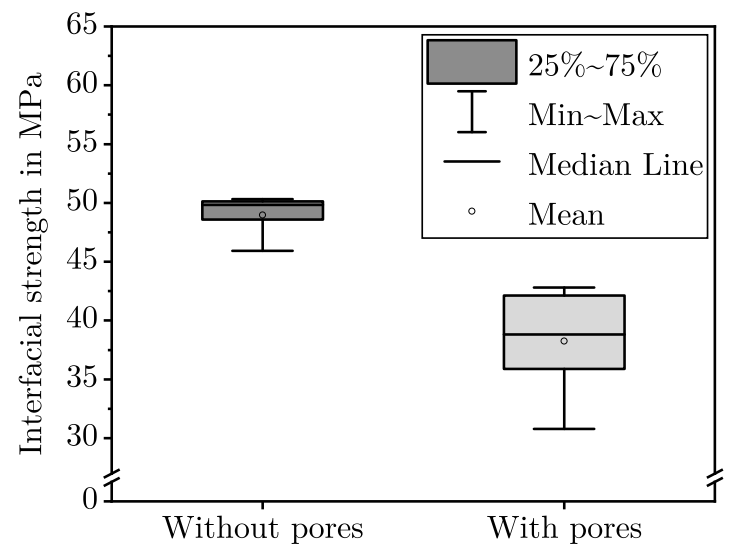

(b)

Fig. 15. Visibility and influence of pores. a: Fracture surfaces of three specimens at a nozzle temperature of $230{ }^{\circ} \mathrm{C}$. The cooling time of the specimens was $18.5 \mathrm{~s}$ and $23.4 \mathrm{~s}$ respectively. Pores can be found in some places on the interface. b: The interfacial strength according to IAM/F for the specimens of the group $230{ }^{\circ} \mathrm{C}$ and $18.5 \mathrm{~s}$ cooling time. The group is separated into specimens with pores and without pores on the interface.

the scatter within the groups would be lower and their mean value would be higher. Thus it is assumed that pores increase scatter and decrease the interfacials strength within a parameter set.

The specimens above $9.2 \mathrm{~s}$ cooling time have in common that they were printed simultaneously and so there had always been travel moves between the individual cuboids. As no pores were present in specimens printed with a cooling time of $3.2 \mathrm{~s}$, one cause can be excluded: pores do not occur due to foaming effects by water enclosed in the polymer. In addition, no correlation between humidity and interfacial strength was found. Thus, the occurrence of pores is related to the travel moves from one cuboid to the next in the printing process. Two effects are connected to those travel moves: oozing and stringing [12]. Oozing is the uncontrolled leakage of polymer from the nozzle when no pressure is applied. If polymer is deposited during a travel move of the printing head, thin polymer strings are drawn, an effect named stringing. Both effects were observed in the study at hand. Both, oozing and stringing, cause a loss of material which is no longer available for the printing. As FFF-technology cannot actively control the flow of material, the missing amount of polymer is not accounted for. The loss of material during extrusion may result in arching the surface. Then, printing the next layer can trap air, thereby causing pores. The arching effect was experimentally verified by printing single tracks on top of each other while the printing process was purposely interrupted and retracted. Figure 16 shows a $\mu \mathrm{CT}$ scan of two tracks printed with interrupt and retract or without both, respectively. In general, the roughness can be attributed to grey-scale gradients of the $\mu \mathrm{CT}$ scan. The track without interrupt and retract has a smooth surface, while the other track show periodic deepenings. In this experiment, the loss of material that would otherwise occur due to oozing has been achieved by the accumulation of material at the beginning of the track. it is very likely that the pores are created by welding on the next track if such deepenings exist on the previous one.

To avoid the phenomena oozing and stringing, a socalled retract is run. For this, the filament is slightly drawn from the nozzle thus reducing the pressure and stopping the material flow. When printing the cuboids used in this experimental study, retracting was used to avoid material 


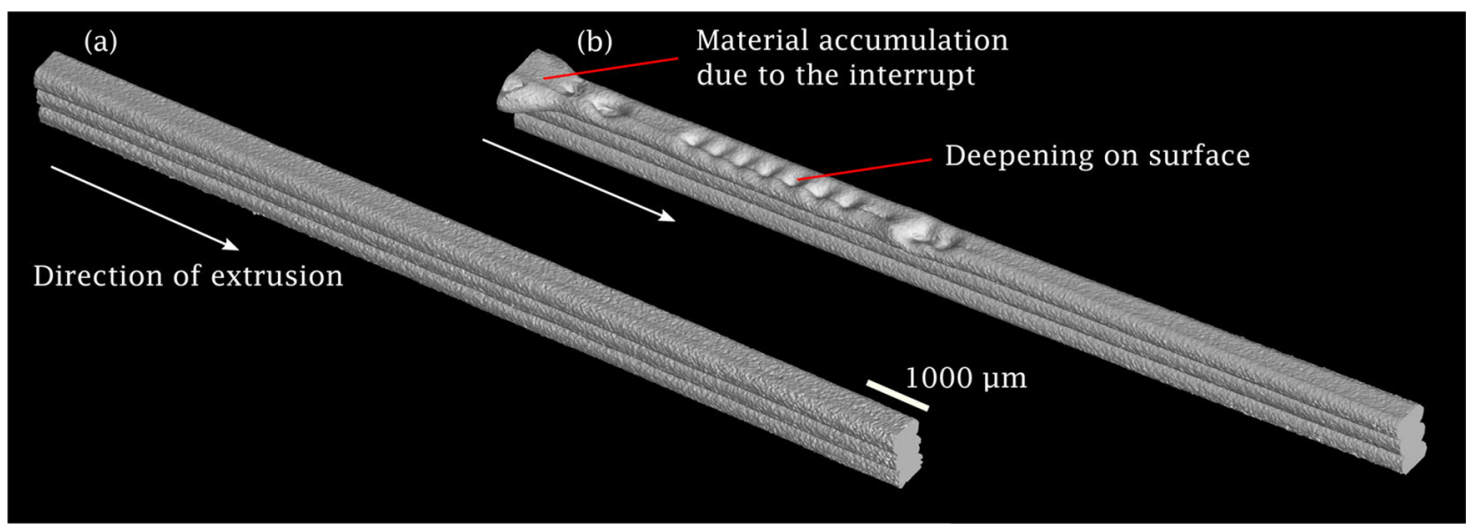

Fig. 16. Two tracks with three layers, both printed at $210^{\circ} \mathrm{C}$. a: This track was produced without an interrupt and retract and there are no surface defects. b: This track was produced with an interrupt and retract. The nozzle remained at the beginning of the track for the interrupt and the retract, which is why there was a small accumulation of material. Deepenings in the surface can be seen along the direction of extrusion.

loss. Nonetheless, with increasing nozzle temperature, retracting cannot fully counteract both phenomena. This is due to the decreased viscosity and explains why the number of specimens with pores increases with increasing nozzle temperature.

In summary, defects such as pores result in a decrease of the interfacial strength and to higher scatter within the test series. The higher the scatter, the less accurate are predictions of the material behaviour based on printing parameters.

\subsection{Appropriate application of the method}

To print and investigate specimens according to realistic production processes, several objects can be printed simultaneously. Here, defects and scatter of the interfacial strength have to be expected. Another application is production-related testing on various plastics to investigate the influence of travel moves. This can also be used to determine the maximum nozzle temperature that can be set for simultaneous printing so that little or no defects can be expected. For the PLA used here, $210^{\circ} \mathrm{C}$ could be specified as the maximum temperature. A single print can take place at a higher nozzle temperature to maximise the interfacial strength. With this, optimal operating parameters in terms of maximal interfacial strength can be defined for filaments. One should keep in mind that even for the same polymer, material properties of printed objects might vary [26]. To obtain more fundamental material insights, specimens should not be printed simultaneously to minimise the effects mentioned in the last chapter.

Regardless of the manufacturing process, a suitable method for measuring interfacial area and strength as to be chosen in accordance with the demanded accuracy. IAM/E shows a minimised measurement effort and can detect large effects such as that of the nozzle temperature. IAM/E is highly suitable when it is more important to measure the strength in relation to the geometry than the actual interfacial strength. However, it is not possible to obtain a definite interfacial strength and to compare it with the bulk material strength. A correction by the ratio $R$ can be run, but nonetheless, that accuracy is still below that obtained applying IAM/F. IAM/I can be used when the focus lies upon the comparison of the fracture surface with all other interfacial areas. If this is not the aim of the investigation, IAM/F should always be used, as this is the more precise method and can also be carried out more quickly compared to IAM/I. Even small effects can be reliably found width $\mathrm{IAM} / \mathrm{F}$. If the interfacial strength is close to the material strength, a correction may be necessary due to the curvature of the fracture surface.

Lastly, the question when to use SWS is addressed here. As mentioned earlier, the procedure eliminates any effects caused by voids between printed layers. As a consequence, multilayer and multidimensional specimens should be used when structural characterstics, such as interlayer voids or track orientation, are to be understood. In contrast, SWS are recommended to identify polymer properties of printed objects. In addition to nozzle temperature and cooling time, other FFF-parameters can be investigated using SWS, as has already been done in some cases [14,17]. One area of application could be the interfacial strength determination for different cooling times in order to adapt models for the prediction of interfacial strengths at different locations in components. Models for predicting the weld strength already exist in part [27].

\section{Conclusion}

Tensile specimens were produced from PLA by using the FFF process with wall widths corresponding to only one extrusion width in order to exclude the influence of voids between printed tracks. These specimens were used to determine the interfacial strength between the layers. The determination of the interfacial area at the fracture location was investigated using three methods based on the extrusion width, the interface width and the fracture surface. This was used to investigate how the methods affect the resulting interfacial strength. It was found that the determination of the interfacial area by measuring the fracture surface is the 
most accurate method and thus the interfacial strength can be determined precisely. It was further shown that determining the interfacial area by measuring the extrusion width of the specimen (IAM/E) is not acceptable for a precise calculation of the interfacial strength.

In order to answer the question whether the interfacial strength can reach the bulk material strength, the bulk material strength was previously determined using extruded strands. With the help of IAM/F and microscope and SEM images of the fracture surface, it could be shown that the interfacial strength at $230{ }^{\circ} \mathrm{C}$ nozzle temperature and $3.2 \mathrm{~s}$ cooling time comes close to the bulk material strength with $96 \%$. It can therefore be assumed that it is possible to achieve the bulk material strength in the FFF process and that in some cases the voids between the sheets are responsible for a reduction in strength.

Furthermore, it was investigated whether the travel moves have an influence on the interfacial strength. It could be shown that oozing occurs due to travel moves in combination with a high nozzle temperature and despite retract, and this can lead to pores at the interface. These pores reduce the interfacial strength and thus increase the scatter within a sample batch. If components are printed simultaneously in the FFF process, then consequently the highest nozzle temperature does not always have to enable the highest possible interfacial strength.

Acknowledgments. This work is funded by the Deutsche Forschungsgemeinschaft (DFG, German Research Foun-dation) project EL 473/10-1 "Additively manufactured foam sandwich structures - a novel approach for the generative processing and characterization of foam based, three-dimensional graded materials for lightweight design". Paula Wesenmann and Theresa Jenne at Karlsruhe Institute of Technology for their support in the experimental study. The discussions with Manuel Morais, Fraunhofer ICT, are highly acknowledged.

\section{Author contributions}

Anselm Heuer: Conceptualization, Methodology, Formal analysis, Investigation, Writing - original draft, Visualization, Project administration. Jonas Huether: Conceptualization, Writing original draft, Visualization. Wilfried V. Liebig: Conceptualization, Resources, Writing - review \& editing, Supervision. Peter Elsner: Resources, Writing - review \& editing, Supervision.

\section{References}

1. J.F. Rodríguez, J.P. Thomas, J.E. Renaud, Mechanical behavior of acrylonitrile butadiene styrene (ABS) fused deposition materials. Experimental investigation, Rapid Prototyp. J. 7 (2001) 148-158

2. S.-H. Ahn, M. Montero, D. Odell, S. Roundy, P.K. Wright, Anisotropic material properties of fused deposition modeling ABS, Rapid. prototyp. J. 8 (2002) 248-257

3. B. Akhoundi, A.H. Behravesh, Effect of filling pattern on the tensile and flexural mechanical properties of FDM 3D printed products, Exp. Mech. 59 (2019) 883-897
4. C. Ziemian, M. Sharma, S. Ziemian, Anisotropic mechanical properties of ABS parts fabricated by fused deposition modelling, Mech. Eng. 23, (2012) 159-180

5. O.S. Es-Said, J. Foyos, R. Noorani, M. Mendelson, R. Marloth, B.A. Pregger, Effect of layer orientation on mechanical properties of rapid prototyped samples, Mater. Manufactur. Process. 15 (2000) 107-122

6. A. Bellini, S. Güçeri, Mechanical characterization of parts fabricated using fused deposition modeling, Rapid Prototyp. J. 9 (2003) 252-264

7. Q. Sun, G.M. Rizvi, C.T. Bellehumeur, P. Gu, Effect of processing conditions on the bonding quality of FDM polymer filaments, Rapid Prototyp. J. 14 (2008) 72-80

8. F. Yang, R. Pitchumani, Healing of thermoplastic polymers at an interface under nonisothermal conditions, Macromolecules 35 (2002) 3213-3224

9. A. García-Domínguez, J. Claver, A.M. Camacho, M.A. Sebastin, Considerations on the applicability of test methods for mechanical characterization of materials manufactured by FDM, Materials 13 (2020) 28

10. G.P. Greeff, M. Schilling, Closed loop control of slippage during filament transport in molten material extrusion, Addit. Manufactur. 14 (2017) 31-38

11. V.E. Kuznetsov, A.N. Solonin, A. Tavitov, O. Urzhumtsev, A. Vakulik, Increasing strength of FFF three-dimensional printed parts by influencing on temperature-related parameters of the process, Rapid Prototyp. J. 26 (2020) 107-121

12. G.P. Greeff, M. Schilling, Single print optimisation of fused filament fabrication parameters, Int. J. Adv. Manufactur. Technol. 99 (2018) 845-858

13. J.Y. Wang, D.D. Xu, W. Sun, S.M. Du, J.J. Guo, G.J. Xu, Effects of nozzle-bed distance on the surface quality and mechanical properties of fused filament fabrication parts, IOP Conf. Ser.: Mater. Sci. Eng. 479 (2019) 012094

14. T.J. Coogan, D.O. Kazmer, Bond and part strength in fused deposition modeling, Rapid Prototyp. 23 (2017) 414-422

15. P. Han, A. Tofangchi, A. Deshpande, S. Zhang, K. Hsu, An approach to improve interface healing in FFF-3D printed Ultem 1010 using laser pre-deposition heating, Proc. Manufactur. 34 (2019) 672-677

16. J. Allum, J. Kitzinger, Y. Li, V.V. Silberschmidt, A. Gleadall, ZigZagZ: improving mechanical performance in extrusion additive manufacturing by nonplanar toolpaths, Addit. Manufactur. 38 (2021) 101715

17. L. Fang, Y. Yan, O. Agarwal, J.E. Seppala, K.J. Hemker, S.H. Kang, Processingstructure-property relationships of bisphenol-A-polycarbonate samples prepared by fused filament fabrication, Addit. Manufactur. 35 (2020) 101285

18. A. Lepoivre, N. Boyard, A. Levy, V. Sobotka, Heat transfer and adhesion study for the FFF additive manufacturing process, Proc. Manufactur. 47 (2020) 948-955

19. J. Huether, P. Rupp, I. Kohlschreiber, K.A. Weidenmann, An enhanced method to determine the Young's modulus of technical single fibres by means of high resolution digital image correlation, Measur. Sci. Technol. 29 (2018) 045601

20. T.J. Coogan, D.O. Kazmer, Healing simulation for bond strength prediction of FDM, Rapid Prototyp. J. 23 (2017) 551-561

21. S. Prager, M. Tirrell, The healing process at polymerpolymer interfaces, J. Chem. Phys. 75 (1981) 5194-5198

22. A.M. Peterson, Review of acrylonitrile butadiene styrene in fused filament fabrication: a plastics engineering-focused perspective, Addit. Manufactur. 27 (2019) 363-371 
23. C. Casavola, A. Cazzato, V. Moramarco, C. Pappalettere, Orthotropic mechanical properties of fused deposition modelling parts described by classical laminate theory, Mater. Des. 90 (2016) 453-458

24. J. Allum, A. Gleadall, V.V. Silberschmidt, Fracture of 3D-printed polymers: crucial role of filament-scale geometric features, Eng. Fract. Mech. 224 (2020) 106818

25. R. Schnell, M. Stamm, C. Creton, Mechanical properties of homopolymer interfaces: Transition from simple pullout to crazing with increasing interfacial width, Macromolecules 32 (1999) 3420-3425

26. J.J.Schwartz, J. Hamel, T. Ekstrom, L. Ndagang, A.J. Boydston, Not all PLA filaments are created equal: an experimental investigation, Rapid Prototyp. J. 26 (2020) 1263-1276

27. S.F. Costa, F.M. Duarte, J.A. Covas, Estimation of filament temperature and adhesion development in fused deposition techniques, J. Mater. Process. Technol. 245 (2017) 167-179

Cite this article as: Anselm Heuer, Jonas Huether, Wilfried V. Liebig, Peter Elsner, Fused filament fabrication: comparison of methods for determining the interfacial strength of single welded tracks, Manufacturing Rev. 8, 32 (2021) 